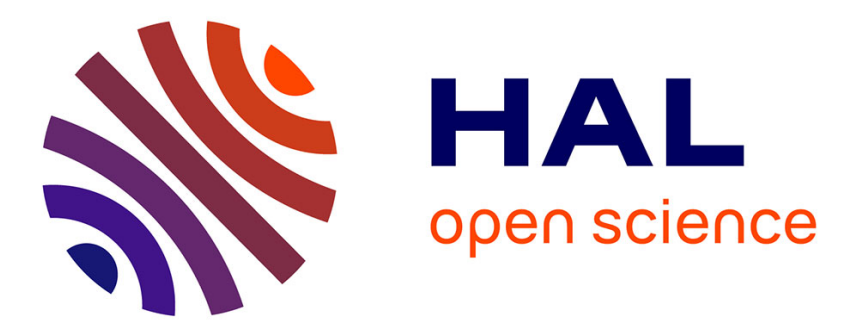

\title{
Rapid and Convenient Synthesis of Original 5-Substituted Quinoli- no[3,4-b]quinoxalin-6(5H)-ones under Eco-Friendly Conditions
}

Omar Khoumeri, François-Xavier Vanelle, Maxime D Crozet, Thierry Terme, Patrice Vanelle

\section{To cite this version:}

Omar Khoumeri, François-Xavier Vanelle, Maxime D Crozet, Thierry Terme, Patrice Vanelle. Rapid and Convenient Synthesis of Original 5-Substituted Quinoli- no[3,4-b]quinoxalin-6(5H)-ones under Eco-Friendly Conditions. SYNLETT, 2016, 27 (10), pp.1547-1550. 10.1055/s-0035-1561577 . hal01406037

\section{HAL Id: hal-01406037 \\ https://hal.science/hal-01406037}

Submitted on 5 Dec 2016

HAL is a multi-disciplinary open access archive for the deposit and dissemination of scientific research documents, whether they are published or not. The documents may come from teaching and research institutions in France or abroad, or from public or private research centers.
L'archive ouverte pluridisciplinaire HAL, est destinée au dépôt et à la diffusion de documents scientifiques de niveau recherche, publiés ou non, émanant des établissements d'enseignement et de recherche français ou étrangers, des laboratoires publics ou privés. 


\section{Rapid and Convenient Synthesis of Original 5-(substituted)quinolino $[3,4-b] q u i n o x a l i n-6(5 H)$-ones under Eco-Friendly Conditions}

\author{
OmarKhoumeri \\ François-XavierVanelle ${ }^{a}$ \\ Maxime D. Crozet ${ }^{\mathrm{a}}$ \\ ThierryTerme ${ }^{a}$ \\ PatriceVanelle*a
}

${ }^{a}$ Aix-Marseille Université, Institut de Chimie Radicalaire ICR, UMR CNRS 7273, Laboratoire de Pharmaco-Chimie Radicalaire, Faculté de Pharmacie, 27 Boulevard Jean Moulin - CS 30064 13385 Marseille Cedex 05, France<smiles>[X]c1cccc(N)c1</smiles><smiles>[X]c1ccc(-n2c(=O)c3nc4ccccc4nc3c3ccccc32)cc1</smiles>

12 examples

\section{Received:
Accepted:
Published onlin
DOI:}

AbstractWe report herein the synthesis of5-(substituted)quinolino[3,4b]quinoxalin-6(5H)-one derivatives 3-(2bromophenyl)quinoxaline-2-carboxylateunderone-pot Buchwald-Hartwig coupling/lactamization reaction.

Quinoline, Aromatic anilines, Buchwald-Hartwig reaction

Key wordsquinoxaline, aromatic anilines, Buchwald-Hartwig reaction, lactamization reaction

\section{Introduction}

Quinoxalines are common structural motifs in many natural and synthetic compounds with interesting biological activities. Many quinoxaline derivatives show a wide range of biological activities,making thempotential antitumor, ${ }^{1}$ antibiotic, ${ }^{2}$ antiviral, ${ }^{3}$ anti-HIV ${ }^{4}$ and anti-inflammatory agents ${ }^{5}$. The list of their uses in medicinal chemistry is long. ${ }^{6}$ The olaquindox (OLA), 2-[(2hydroxyethyl)carbamoyl]-3-methylquinoxaline 1,4-dioxide, presents strong antimicrobial activities and is used in subtherapeutics (animal feed)topromote animal growth. ${ }^{7}$ Quinacillin is significantly active $(0.15-0.62 \mu \mathrm{g} / \mathrm{mL}, 0.32-1.35 \mu \mathrm{M})$ against Staphylococcus aureus. ${ }^{8}$

Thephenanthridin groupis present in many bioactive natural and pharmaceutical products. ${ }^{9} \quad$ They show particularly interestingbiological activity as anticancer agents. ${ }^{10}$ In order to synthesizestructures with high biological activitypotential, we combined the quinoxaline moiety with the phenanthridinmoiety. Furthermore, to the best of our knowledge, few syntheses of quinolino[3,4-b]quinoxalin-6(5H)-one derivatives have been reported $^{11}$ and none used coupling reactions.
Palladium-catalyzed coupling reactions have been extensively used in medicinal chemistry.The synthesis process is not expensive and possesses a widecompatibility with many functional groups.This approachis an effective way of functionalizing the quinoxaline derivatives as Suzuki-Miyaura, ${ }^{12}$ Sonogashira ${ }^{13}$ or Buchwald-Hartwig ${ }^{14}$ reactions, and the 2-bromophenylboronicacid (2)proved promising as a substrate for coupling/lactamization reaction. ${ }^{15}$

As part of our research program to develop new bioactive compounds, ${ }^{16}$ we report herein the first one-pot Buchwald-Hartwig coupling/lactamization reaction in a quinoxalineseries for the synthesis of 5-(substituted)quinolino[3,4-b]quinoxalin-6(5H)-ones.

The startingproduct,for one-pot coupling/lactamizationreaction, ethyl 3-(2-bromophenyl)quinoxaline-2-carboxylate $\quad \mathbf{3}$,was synthesized by Suzuki-Myaura reaction between ethyl 3chloroquinoxaline-2-carboxylate (1) and 2-bromophenylboronicacid (2) using 0.02 equiv of $\mathrm{Pd}\left(\mathrm{PPh}_{3}\right)_{4}$ as a palladium source and 5equivof $\mathrm{Na}_{2} \mathrm{CO}_{3}$ asa base in anacetonitrile-water mixture (3/1) at $100{ }^{\circ} \mathrm{C}$ for $5 \mathrm{~h}($ Scheme 1$)$. The key product 3 was obtained in good yields $(73 \%) .{ }^{17}$

Scheme 1 Preparation of ethyl 3-(2-bromophenyl)quinoxaline-2carboxylate (3)

Our initial strategy consisted in the introduction of the amide group from ester,firstreplacingthe ethoxy group, which wasfollowed byan intramolecular Bulwald-Hartwig reaction/cyclization. The amidation reaction was tested with starting product $\mathbf{3}$ and aniline in presence of $t$-BuOK at room temperature in toluenefor $30 \mathrm{~min}$. The formation of intermediate 3-(2-bromophenyl)- $N$-phenylquinoxaline2-carboxamide was confirmed by TLC and LCMS.Then, we applied the coupling reaction conditionswith different catalysts $\left(\mathrm{Pd}(\mathrm{OAc})_{2}, \mathrm{Pd}\left(\mathrm{PPh}_{3}\right)_{4}\right)$, in presence of bases $\left(\mathrm{K}_{2} \mathrm{CO}_{3}, \mathrm{Cs}_{2} \mathrm{CO}_{3}, t\right.$ $\mathrm{BuOK})$ at $110^{\circ} \mathrm{C}$. The best yield $(44 \%)$ of 5-phenylquinolino[3,4$b$ ]quinoxalin-6(5H)-one (5a) was obtained with palladium acetate, BINAP as supporting ligand and $\mathrm{Cs}_{2} \mathrm{CO}_{3}$ at $110^{\circ} \mathrm{C}$ for $12 \mathrm{~h}$.

In view of these last poor yields, a simpler strategy was developed,only involving coupling reaction conditions with ethyl 3- 
(2-bromophenyl)quinoxaline-2-carboxylate (3). Preliminary tests wereconducted with ethyl 3-(2-bromophenyl)quinoxaline-2carboxylate (3) and aniline withdifferent catalysts $\left[\mathrm{Pd}(\mathrm{OAc})_{2}\right.$, $\left.\mathrm{Pd}\left(\mathrm{PPh}_{3}\right)_{4}\right]$, in the presence of base $\left(\mathrm{K}_{2} \mathrm{CO}_{3}, \mathrm{Cs}_{2} \mathrm{CO}_{3}, t\right.$-BuOK) at $110^{\circ}$ Cin toluene (Table 1$)$
A better yield was obtained with palladium acetate as catalyst and BINAP as ligand in presence of $\mathrm{Cs}_{2} \mathrm{CO}_{3}$ in toluene at $110^{\circ} \mathrm{C}$ for $5 \mathrm{~h}$ (97\% yield).

Scheme 2 Reaction of ethyl 3-(2-bromophenyl)quinoxaline-2-carboxylate (3) and aniline (4a)

Table 1Optimization reaction between ethyl 3-(2-bromophenyl)quinoxaline-2-carboxylateand aniline ${ }^{\mathrm{a}}$

\begin{tabular}{|c|c|c|c|c|c|}
\hline Entry & Catalyst $^{\mathbf{a}}$ & Liganda $^{a}$ & Base $^{a}$ & Time (h) & Yield $^{\mathrm{b}}(\%)$ \\
\hline 1 & $\mathrm{Pd}(\mathrm{OAc})_{2}$ & BINAP & $\mathrm{K}_{2} \mathrm{CO}_{3}$ & 15 & 78 \\
\hline 2 & $\mathrm{Pd}(\mathrm{OAc})_{2}$ & BINAP & $t$-BuOk & 15 & Complex mixture \\
\hline 3 & $\mathrm{Pd}(\mathrm{OAc})_{2}$ & BINAP & $\mathrm{Cs}_{2} \mathrm{CO}_{3}$ & 5 & 97 \\
\hline 4 & $\mathrm{Pd}\left(\mathrm{PPh}_{3}\right)_{4}$ & - & $\mathrm{K}_{2} \mathrm{CO}_{3}$ & 10 & Starting material \\
\hline
\end{tabular}
based on substrate 3 .

\begin{tabular}{lcc}
\hline Aromatic amine & Product $^{\mathrm{a}}$ & Yield $^{\mathrm{b}}(\%)$ \\
\hline Aniline & $\mathbf{5 a}$ & 97 \\
2-Chloroaniline & $\mathbf{5 b}$ & 63 \\
3-Chloroaniline & $\mathbf{5 c}$ & 80 \\
3-Fluoroaniline & $\mathbf{5 d}$ & 72 \\
3-(Trifluoromethyl)aniline & $\mathbf{5 e}$ & 65 \\
3-Aminobenzonitrile & $\mathbf{5 f}$ & 82 \\
4-Chloroaniline & $\mathbf{5 g}$ & 82 \\
4-Fluoroaniline & $\mathbf{5 h}$ & 88 \\
4-Methoxyaniline & $\mathbf{5 i}$ & $17(50)^{\mathrm{c}}$ \\
3,4-Chloroaniline & $\mathbf{5 j}$ & $50(60)^{\mathrm{c}}$ \\
2,3-Chloroaniline & $\mathbf{5 k}$ & 76 \\
p-Toluidine & $\mathbf{5 l}$ & 90 \\
\hline
\end{tabular}

Scheme 3 Synthesis of 5-(substituted)quinolino[3,4-b]quinoxalin$6(5 H)$-ones5a-l

Table 2 Generalization of one-pot coupling/lactamization reactions

a All the reactions are performed using 1.5 equiv of aniline, $20 \mathrm{~mol} \%$ of $\mathrm{Pd}(\mathrm{OAc})_{2}, 15 \mathrm{~mol} \%$ of BINAP, 2.2 equiv of $\mathrm{Cs}_{2} \mathrm{CO}_{3}$ in toluene for $5 \mathrm{~h}$ in $110^{\circ} \mathrm{C}$. All yields refer to chromatographically isolated pure products based
CThe reactions are performed using $20 \mathrm{~mol} \%$ ofPd(OAc) 2,15 mol\% of BINAP, 3 equiv of $\mathrm{K}_{2} \mathrm{CO}_{3}$ in toluene for $24 \mathrm{~h}$ in $110^{\circ} \mathrm{C}$

After optimization of the conditions (Table 1, entry3)we generalized the coupling/lactamization reaction with different aniline derivatives and the corresponding 5(substituted)quinolino[3,4- $b$ ]quinoxalin-6(5H)-one derivatives were obtained in good yields $(50-97 \%$ Scheme 3, Table 2).Very good yields were obtained with no substituted aniline $(\mathbf{5 a}$ 97\%) and $p$ toluidine $(\mathbf{5 1 9 0 \% )}$. Good yields were obtainedwith anilines substituted by halogen atom in ortho, meta or paraposition (63$88 \%$ ). A yield of $82 \%$ was obtained with anilines substituted by $\mathrm{CN}$ or $65 \%$ yield with $\mathrm{CF}_{3}$ groupin metaposition. With dichlorinatedanilines, moderate to good yields $(\mathbf{5 j} 60 \%$ and $\mathbf{5 k} 76 \%)$ were obtained. No reaction was observed with anilines substituted by nitro or cyano group in para position.Finally, a low yield was obtained with 4-methoxyaniline $\left(\mathbf{5 i} 17 \%\right.$ with $\mathrm{Cs}_{2} \mathrm{CO}_{3}$ for5h and $50 \%$ with $\mathrm{K}_{2} \mathrm{CO}_{3}$ for $24 \mathrm{~h}$ ).

In conclusion, we developed a very efficient one-pot reaction combining Buchwald-Hartwig and lactamization reactions for the synthesis of quinolino[3,4- $b$ ]quinoxalin-6(5H)-onederivatives. Starting material ethyl 3-(2-bromophenyl)quinoxaline-2-carboxylate (3) was prepared by Suzuki-Miyaura reaction from ethyl 3chloroquinoxaline-2-carboxylate and 2-bromophenylboronic acid. The pharmacological evaluation of these highly potential bioactive compounds is under active investigation.

\section{Acknowledgment}

This work was supported by the Centre National de la RechercheScientifique, Aix-Marseille. We express our thanks to V. Remusat for recording ${ }^{1} \mathrm{H}$ NMR and ${ }^{13} \mathrm{C}$ NMR spectra and $\mathrm{T}$. Schembri for recording mass spectra. 


\section{References and Notes}

(1) (a)Monge, A.; Martinez-Crespo, F. J.; Lopez de Cerain, A.; Palop, J. A.; Narro, S.; Senador, V.; Marin, A.; Sainz, Y.; Gonzalez, M.; Hamilton, E.; Barker, A. J. J. Med. Chem. 1995, 38, 4488. (b) Lindsley, C. W.; Zhao, Z.; Leister, W. H.; Robinson, R. G.; Barnett, S. F.; Defeo Jones, D.; Jones, R. E.; Hartman, G. D.; Huff, J. R.; Huber, H. E.; Duggan, M. E. Bioorg. Med. Chem. Lett. 2005, 15, 761.(c) Marcus, L.; Kingi, N.; Bergman, J. J. Med. Chem. 2008, 51, 7744. (d) Lee, S.-H.; Kim, N.; Kim, S.-J.; Song, J.; Gong, Y.-D.; Kim, S.-Y. J. Cancer Res. Clin. Oncol. 2013, 139,1279.

(2) (a)Shoji, J.;Katakiri, K. J. Antibiotics Ser. A 1961, 14, 335. (b)Kim, Y. B.; Kim, Y. H.; Park, J. Y.; Kim, S. K.Bioorg. Med. Chem. Lett.2004,14, 541 .

(3) (a) Sehlsedt, U.; Aich, P.; Bergman, J.; Vallberg, H.; Norden, B.; Graslund, A. J. Mol. Biol. 1998, 278, 31. (b) Harmenberg, J.; Akesson-Johansson, A.; Graslund, A.; Malmfors, T.; Bergman, J.; Wahren, B.; Akerfeldt, S.; Lundblad, L.; Cox, S.Antiviral Res. 1991,15, 193. (c) Wilhelmsson, L. M.; Kingi, N.; Bergman, J. J. Med. Chem. 2008, 51, 7744.

(4) (a) Munos, M.-H.; Mayrargue, J.; Fournet, A.; Gantier, J.-C.; Hocquemiller, R.; Moskowitz, H. Chem. Pharm. Bull.1994, 42, 1914. (b)Maga, G.; Gemma, S.; Fattorusso, C.; Locatelli, G. A.; Butini, S.; Persico, M.; Kukreja, G.; Romano, M. P.; Chiasserini, L.; Savini, L.; Novellino, E.; Nacci, V.; Spadari, S.; Campiani, G.Biochemistry2005, 44, 9637 .

(5) (a)Badran, M.M.; Abouzid, K.A.M.; Hussein, M.H.M. Arch. Pharm. Res.2003, 26, 107.(b) Kim, Y. B.; Kim, Y. H.; Park, J. Y.; Kim, S. K. Bioorg. Med. Chem. Lett.2004, 14, 541. (c) He, W.; Meyers, M. R.; Hanney, B.; Sapada, A.; Blider, G.; Galzeinski, H.; Amin, D.; Needle, S.; Page, K.; Jayyosi, Z.; Perrone, H. Bioorg. Med. Chem. Lett. 2003, 13, 3097. (d) Sakata, G.; Makino, K.; Kurasawa, Y. Heterocycles 1988, 27, 2481. (e) Khan, S. A.; Mullick, P.; Pandit, S.; Kaushik, D. Acta Pol. Pharm. 2009, 66, 169. (f) Abu-Hashem, A-A.; Gouda, M. A.; Badria, F. A. Eur. J. Med. Chem.2010, 45, 1976.

(6) Cheeseman, G.W.H.; Cookson, R.F.The Chemistry of Heterocyclic Compounds; Weissberger, A., Taylor, E.C., Eds.; John Wiley and Sons: New York, NY, USA, 1979,35, 1.

(7) (a) Carta, A., Corona, P., Loriga, M.Curr. Med. Chem. 2005, 12, 2259.(b) Tianci, X.; Xingjia, G.; Lei, Z.; Fang, P.; Junna, L.; Yunyu Z.; Hongjing, J.Food. Chem. Toxicol.2012,50, 2540.

(8) Hugo, W. B.; Stretton, R. G Nature 1964, 202, 1217.

(9) (a)Michael, J. P. Nat. Prod. Rep.,1995, 12, 465. (b) Li, J.-H.; Serdyuk, L.; Ferraris, D. V.; Xiao, G.; Tays, K. L.; Kletzly, P. W.; Li, W.; Lautar, S.; Zhang, J.; Kalish, V. J. Bioorg. Med. Chem. Lett. 2001, 11, 1687. (c) Ishida, J.; Yamamoto, H.; Kido, Y.; Kamijo, K.; Murano, K.; Miyake, H.; Ohkubo, M.; Kinoshita, T.; Warizaya, M.; Iwashita, A.; Mihara, K.; Matsuokac, N.; Hattori, K. Bioorg. Med. Chem. 2006, 14, 1378. (d) Patil, S. Kamath, S. Sanchez, T. Neamati, N. Schinazi R. F. and Buolamwini, J. K. Bioorg. Med. Chem.2007, 15, 1212 (e) Jin, Z. Nat. Prod. Rep.2009, 26, 363. (f) Lehtio, L.; Jemth, A.-S.; Collins, R.; Loseva, O.; Johansson, A.; Markova, N.; Hammarstro, M.; Flores, A.; HolmbergSchiavone, L.; Weigelt, J.; Helleday, T.; Schüler, H.; Karlberg, T. J. Med. Chem. 2009, 52, 3108. (g) Gui, Q.; Yang, Z.; Chen, X.; Liu, J.; Tan, Z.; Guo, R.; Yu, W. Synlett2013, 24, 1016.(h) Pimparkar, S.; Jeganmohan, M. ChemComm.2014, 50, 12116. (i) Yang, Y.; Huang, H.; Wu, L.; Yun, L. Org.Biomol.Chem. 2014, 12, 5351 .

(10) (a) Poschenrieder, H.; Stachel, H.-D.; Wiesend, B.; Polborn, K. J. Heterocyclic. Chem. 2003, 40, 61. (b) El-Gogary, S. R.; Waly, M. A.; Ibrahim, I. T.; El-Sepelgy, O. Z. Monatsh. Chem.2010, 141, 1253.

(11) Mohamed, E. A.; Gabr, Y.; Ismail, M. M.; Abass, M. An. Quím. $1995,91,579$

(12) (a) Mao, L.; Sakurai, H.; Hirao, T. Synthesis, 2004,15, 2535. (b) Ali, I.; Siyo, B.; Al-Soud, Y.; Villinger, A.; Langer, P. Synthesis2012, 44, 1637 .
(13) (a) Armengol, M.; Joule, J. A. J. Chem. Soc., Perkin Trans. 2001, 1, 978. (b) Kang, F.-A.; Lanter, J. C.; Cai, C.; Sui, Z.; Murray, W. V. Chem. Commun.2010, 46, 1347. (c) Shi, C.; Aldrich, C. C. Org. Lett. 2010,12, 2286.(c) Keivanloo, A.; Bakherad, M.; Rahimi, A.; Taheri, S. A. N. Tetrahedron Lett. 2010, 51, 2409. (d) Gulevskaya, A. V.; Lazarevich, R. Y.; Pozharskii,A. F. Tetrahedron2013, 69, 910. (e) Nakhi, A.; Rahman, M. S.; Seerapu, G. P. K.; Banote, R. K.; Kumar, K. L.; Kulkarni, P.; Haldar, D.; Pal, M. Org. Biomol. Chem., 2013, 11, 4930.(f) Kolli, S. K.; Nakhi, A.; Medishetti, R.; Yellanki, S.; Kulkarni, P.; Ramesh Raju, R.; Pal, M. Bioorg. Med. Chem. Lett.2014, 24, 4460.

(14) Hung, T. Q.; Hoang, D. H.; Thang, N. N.; Dang, T. T.; Ayub, K.; Villinger, A.; Friedrich, A.; Lochbrunner, S.; Flechsig, G.U.; Langer, P. Org. Biomol. Chem.2014, 12, 6151.

(15) (a) Bolibrukh, K.; Khoumeri, O.; Polovkovych, V.; Terme, T.; Vanelle, P. Synlett2014, 25, 2765. (b) Do, H.H.; Tran, H.Q.; Ohlendorf, L.; Ngo, T.N.; Dang, T.T.; Ehlers, P.; Villinger, A.; Langer, P. Synlett2015, 26, 2429

(16) (a) Crozet, M. P.; Gellis, A.; Pasquier, C.; Vanelle, P.; Aune, J.-P. Tetrahedron Lett.1995, 36, 525. (b) Montana, M.;Terme, T.; Vanelle, P.Tetrahedron Lett.2006, 47, 6573. (c) Dunn, L.A.; Burgess, A. G.; Krauer, K. G.; Eckmann, L.; Vanelle, P.; Crozet, M. D. Gillin, F. D.; Upcroft, P.; Upcroft, J. A. Int. J. Antimicrob. Agents2010, 36, 37. (d)Kieffer, C.; Cohen, A.; Verhaeghe, P.; Paloque, L.; Hutter, S.; Castera-Ducros, C.; Laget, M.; Rault, S.; Valentin, A.; Rathelot, P.; Azas, N.; Vanelle, P. Bioorg. Med. Chem.2015, 23, 2377.

(17) Ethyl 3-(2-bromophenyl)quinoxaline-2-carboxylate (3)

To a mixture of ethyl 3-chloroquinoxaline-2-carboxylate (1g, $4.22 \mathrm{mmol})$ and 2-bromophenylboronic acid $(0.93 \mathrm{~g}, 4.64 \mathrm{mmol})$ in acetonitrile $(30 \mathrm{~mL})$, was added $\mathrm{Pd}\left(\mathrm{PPh}_{3}\right)_{4}(0.097 \mathrm{~g}, 0.084 \mathrm{mmol})$ and $2 \mathrm{M}$ aqueous sodium carbonate solution $(10 \mathrm{~mL})$. The resulting mixture was stirred at $100^{\circ} \mathrm{C}$ for $5 \mathrm{~h}$. After cooling the mixture was diluted with dichloromethane and washed 3 times with water and dried over $\mathrm{MgSO}_{4}$. After evaporation, the crude product was purified by silica gel chromatography with $\mathrm{CH}_{2} \mathrm{Cl}_{2}$ as eluantfurnished the product $\mathbf{3}$ as yellow solid;yield: $1.1 \mathrm{~g}(73 \%)$; $\mathrm{mp} 100^{\circ} \mathrm{C} ;{ }^{1} \mathrm{H}$ NMR $\left(250 \mathrm{MHz}, \mathrm{CDCl}_{3}\right) \delta=1.19(\mathrm{t}, J=7.1 \mathrm{~Hz}, 3 \mathrm{H}$, $\left.\mathrm{CH}_{3}\right), 4.33\left(\mathrm{q}, J=7.1 \mathrm{~Hz}, 2 \mathrm{H}, \mathrm{CH}_{2}\right), 7.33-7.40\left(\mathrm{~m}, 1 \mathrm{H}, \mathrm{H}_{\mathrm{Ar}}\right), 7.45-$ $7.85\left(\mathrm{~m}, 2 \mathrm{H}, \mathrm{H}_{\mathrm{Ar}}\right), 7.67\left(\mathrm{~d}, J=8.0 \mathrm{~Hz}, 1 \mathrm{H}, \mathrm{H}_{\mathrm{Ar}}\right), 7.89-7.93(\mathrm{~m}, 2 \mathrm{H}$, $\left.\mathrm{H}_{\mathrm{Ar}}\right), 8.19-8.23\left(\mathrm{~m}, 1 \mathrm{H}, \mathrm{H}_{\mathrm{Ar}}\right), 8.31-8.35\left(\mathrm{~m}, 1 \mathrm{H}, \mathrm{H}_{\mathrm{Ar}}\right) .{ }^{13} \mathrm{C} \mathrm{NMR}$ $\left(62.5 \mathrm{MHz}, \mathrm{CDCl}_{3}\right) \delta=13.7,62.3,122.1,127.6,129.3,130.0,130.3$, 130.5, 131.0, 132.1, 132.3, 139.8, 140.4,142.0, 144.4, 153.2, 164.7. Anal.Calcd for $\mathrm{C}_{17} \mathrm{H}_{13} \mathrm{BrN}_{2} \mathrm{O}_{2}$ : C, 57.16; H, 3.67; N, 7.84. Found: C, 57.61; H, 3.61. N, 7.86.

(18) General procedure of one-potBuchwaldHartwig/lactamizationreactions(5a-l)

A mixture of ethyl 3-(2-bromophenyl)quinoxaline-2-carboxylate (3) (50 mg, $0.14 \mathrm{mmol})$, aniline $(0.21 \mathrm{mmol}, 1.5$ equiv) in toluene $(3 \mathrm{~mL})$, $\mathrm{Cs}_{2} \mathrm{CO}_{3}(100 \mathrm{mg}, 0.3 \mathrm{mmol}), \mathrm{Pd}(\mathrm{OAc})_{2}(6.2 \mathrm{mg}, 0.028 \mathrm{mmol})$, BINAP (14 mg, $0.021 \mathrm{mmol}$ ) was added to a $4 \mathrm{~mL}$ sealed vial. The resulting mixture was stirred at $110^{\circ} \mathrm{C}$ for $5 \mathrm{~h}$. After cooling the mixture was diluted with dichloromethane, washed with $\mathrm{H}_{2} \mathrm{O}(3 \times 40$ $\mathrm{mL}$ ) and dried over $\mathrm{MgSO}_{4}$. After evaporation, the crude product was purified by silica gel chromatography $\left(\mathrm{CH}_{2} \mathrm{Cl}_{2} /\right.$ diethylether: 97/3).

5-phenylquinolino[3,4-b]quinoxalin-6(5H)-one (5a) yellow solid; $\mathrm{mp} 240^{\circ} \mathrm{C} ;{ }^{1} \mathrm{H}$ NMR $\left(250 \mathrm{MHz}, \mathrm{CDCl}_{3}\right) \delta=6.73(\mathrm{~d}, J=7.9 \mathrm{~Hz}$, $\left.1 \mathrm{H}, \mathrm{H}_{\mathrm{Ar}}\right) ; 7.38-7.46\left(\mathrm{~m}, 4 \mathrm{H}, \mathrm{H}_{\mathrm{Ar}}\right) ; 7.59-7.70\left(\mathrm{~m}, 3 \mathrm{H}, \mathrm{H}_{\mathrm{Ar}}\right) ; 7.87-$ $8.01\left(\mathrm{~m}, 2 \mathrm{H}, \mathrm{H}_{\mathrm{Ar}}\right) ; 8,33\left(\mathrm{~d}, J=8.4 \mathrm{~Hz}, 1 \mathrm{H}, \mathrm{H}_{\mathrm{Ar}}\right) ; 8.48(\mathrm{~d}, J=8.5$ $\left.\mathrm{Hz}, 1 \mathrm{H}, \mathrm{H}_{\mathrm{Ar}}\right)$; 9.05-9.08 $\left(\mathrm{m}, 1 \mathrm{H}, \mathrm{H}_{\mathrm{Ar}}\right) .{ }^{13} \mathrm{C} \mathrm{NMR}(62.5 \mathrm{MHz}$, $\left.\mathrm{CDCl}_{3}\right) \delta=116.9,119.6,123.5,125.8,129.0,129.2,130.4,130.4$, $130.7,131.0,131.9,132.9,137.6,140.5,143.1,144.5,145.7$, 160.3. Anal.Calcd for $\mathrm{C}_{21} \mathrm{H}_{13} \mathrm{~N}_{3} \mathrm{O}$ : C, 78.00; H, 4.05; N, 13.00. Found: C, 78.12; H, 4.00. N, 12.91. 
